\title{
The Relationship Between the Chemical Structure of Poly(alkylene glycol)s and Their Aerobic Biodegradability in an Aqueous Environment
}

\author{
Elżbieta Beran · Sylwia Hull $\cdot$ Mieczysław Steininger
}

Published online: 24 April 2012

(C) The Author(s) 2012. This article is published with open access at Springerlink.com

\begin{abstract}
The relationship between the chemical structure of poly(alkylene glycol)s (PAGs) and their biodegradability was studied using a set of polymeric fluids that included poly(ethylene glycol), poly(propylene glycol) (PPG), random copolymers of ethylene oxide (EO) and propylene oxide $(\mathrm{PO})$ differing in the EO/PO ratio as well as PAGs capped with ether or acyl moieties. The PAGs that were tested had an average molecular weight (MW) in the range of $350-3,600 \mathrm{Da}$ and differed in their polymer backbones by either linear (diol type) or branched (triol type) molecules. The ultimate biodegradability of the PAGs was determined according to ISO $14593\left(\mathrm{CO}_{2}\right.$ headspace test) with a non-pre-exposed (as in OECD 310 test) and pre-exposed (adapted) inoculum. PAGs with the structure of PPG and copolymers of EO/PO of diol or triol structures with average molecular weights lower than 1,000 Da can be considered as readily biodegradable. Their ultimate biodegradation exceeds the limit of $60 \%$ (according to the criteria of the OECD 310 test). PAGs with a copolymer structure and MW values ranging between 1,000 and 3,600 Da are not readily biodegradable, but they can be considered as those of inherent ultimate biodegradability. The increased EO content in PAG structures and the acylation of the terminal hydroxyl groups with carboxylic acids favourably influenced their biodegradability. Capped PAGs containing terminal ether groups appeared to be resistant to biodegradation.
\end{abstract}

E. Beran $(\bowtie) \cdot S$. Hull $\cdot$ M. Steininger

Division of Chemistry and Technology of Fuel, Faculty

of Chemistry, Wrocław University of Technology,

ul. Gdańska 7/9, 50-344 Wrocław, Poland

e-mail: elzbieta.beran@pwr.wroc.pl
Keywords Biodegradation - Polyalkylene glycol · PAG · PPG $\cdot$ Capped PAG $\cdot$ ISO 14593

\section{Introduction}

The term poly(alkylene glycol)s (PAGs), along with the terms used in the pertinent literature, i.e., polyglycols, polyethers and poly(alkylene oxides), encompasses a group of synthetic compounds obtained by polymerising alkylene oxides with an epoxide structure. PAGs are predominantly synthesised by using ethylene and propylene oxides, while the use of butylene and higher olefinic oxides is less popular $[1,2]$.

The most popular group of PAG-type compounds (differing in molecular weights, properties and applications) comprises homopolymers of ethylene oxide or propylene oxide as well as random or block copolymers of ethylene and propylene oxides. The molecular structure of PAGs depends not only on the type and proportion of alkylene oxides but also on the type of initiator molecule (a mono-, di- or polyfunctional molecule possessing more than 2 labile hydrogen atoms) used for polymerisation. The initiator influences the number of terminal hydroxyl groups in the PAG molecule and the type of polymer chain, which can be linear (mono-ol and diol-type) or branched (polyoltype). The structures of some of the PAGs are shown in Fig. 1.

In the past two decades, poly(ethylene glycol)s (PEGs), poly(propylene glycol)s (PPGs) and copolymers of ethylene and propylene oxides have been widely used not only for the production of surfactants, polyurethanes, cosmetics and pharmaceuticals but also for manufacturing lubricants [3-5]. It has been observed that there is a growing interest in synthetic oily fluids for the production of lubricants [6]. 
Fig. 1 Type of PAG structures manufactured via polymerisation of ethylene oxide and/or propylene oxide

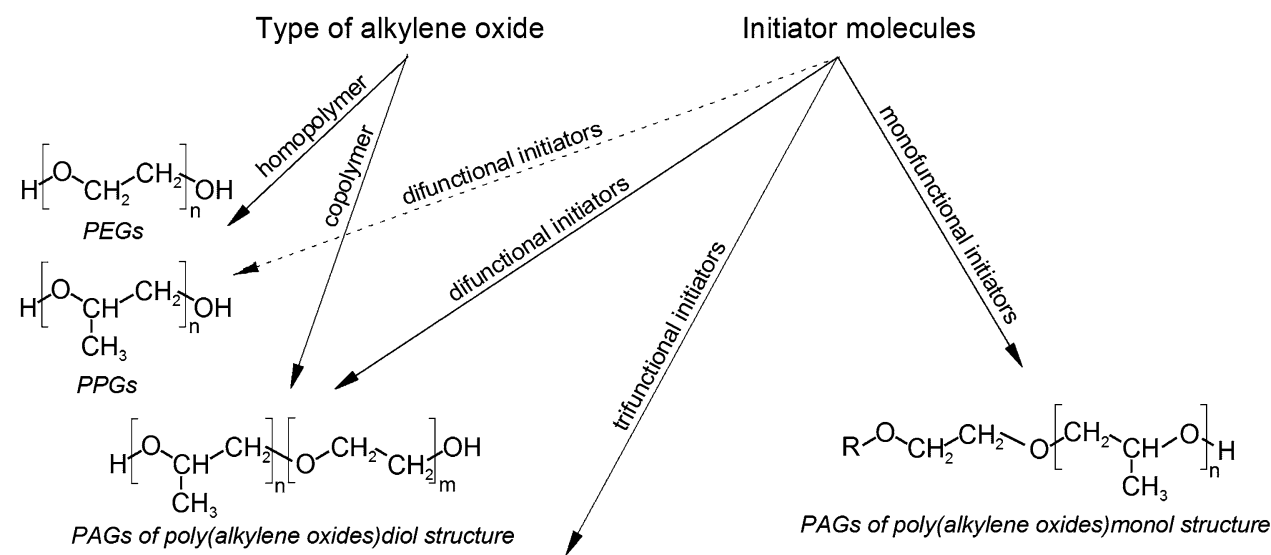

Compared with the lubricating materials manufactured from conventional mineral oils, the products obtained from a wide variety of synthetic fluids display not only better properties but in many instances may also be enriched with unique properties. The enhanced awareness of developing environmentally sustainable technologies and the requirement to assess the environmental impact of chemical substances (including lubricants) have resulted in the advent of interest in ester fluids and fluids with a poly(alkylene glycol) structure. Thus, the use of these oils as substitutes for mineral oils shows a steady upward trend because the resulting lubricants are characterised by better biodegradability in the natural environment and lower ecotoxicity [7].

Noticeably less information has been published on assessing the biodegradability of PAG fluids in comparison with hydrocarbon and ester fluids [8-10]. However, in the past two decades, investigations have been reported that are devoted to the assessment of PEG biodegradability, yet rarely do they address PPG biodegradability [11-17]; these investigations have also reported on the determination of mechanisms that rule PEG or PPG biodegradation. There are only a few reports on the biodegradability of copolymeric PAGs, and there is a lack of data on its dependence on the chemical structure of these polymers $[1,18,19]$.

The aim of the tests performed within the present study was to determine how the structures of PAGs influence their biodegradability, especially the roles of copolymeric structures, average molecular weight and the conversion of their terminal hydroxyl groups leading to polymers capped with ether or acyl groups.

\section{Materials and Methods}

\section{PAG Fluids Tested}

Table 1 characterises the PAGs for which the ultimate aerobic biodegradability in an aqueous medium was established by using standard tests.

PAGs (either of a homopolymeric structure or random copolymers of ethylene oxide and propylene oxide) were chosen to represent a large group of the PAG fluids produced by PCC Rokita SA, Brzeg Dolny, Poland and marketed under the brand names Polikol and Rokopol. The PAGs that were tested differed in their average molecular weights and chemical structures, which is attributable to the various proportions of ethylene oxide and propylene oxide that were used for polymerisation. Depending on the initiator used, the PAGs displayed molecules either of a linear (diol) or a branched (triol) structure (Fig. 1).

The set of the PAGs that was studied also included capped PAGs with terminal hydroxyl groups converted to ester or ether groups. Two of them contain acyl group at the ends of their molecular backbones, with linear PPG 7P diester being diacylated with $n$-hexanoic acid and branched PAG TG $600 \mathrm{E}$ triester acylated with $n$-octanoic acid. Acylations were carried out according to a previously described procedure [19]. The third capped derivative, PPG 7P diMe (dimethyl ether), was obtained in the reaction of PPG 7P with dimethylsulfate according to the literature [20].

The parameters characterising studied classes of oily fluids, such as kinematic viscosity, viscosity index and pour point are given in Table 1. 


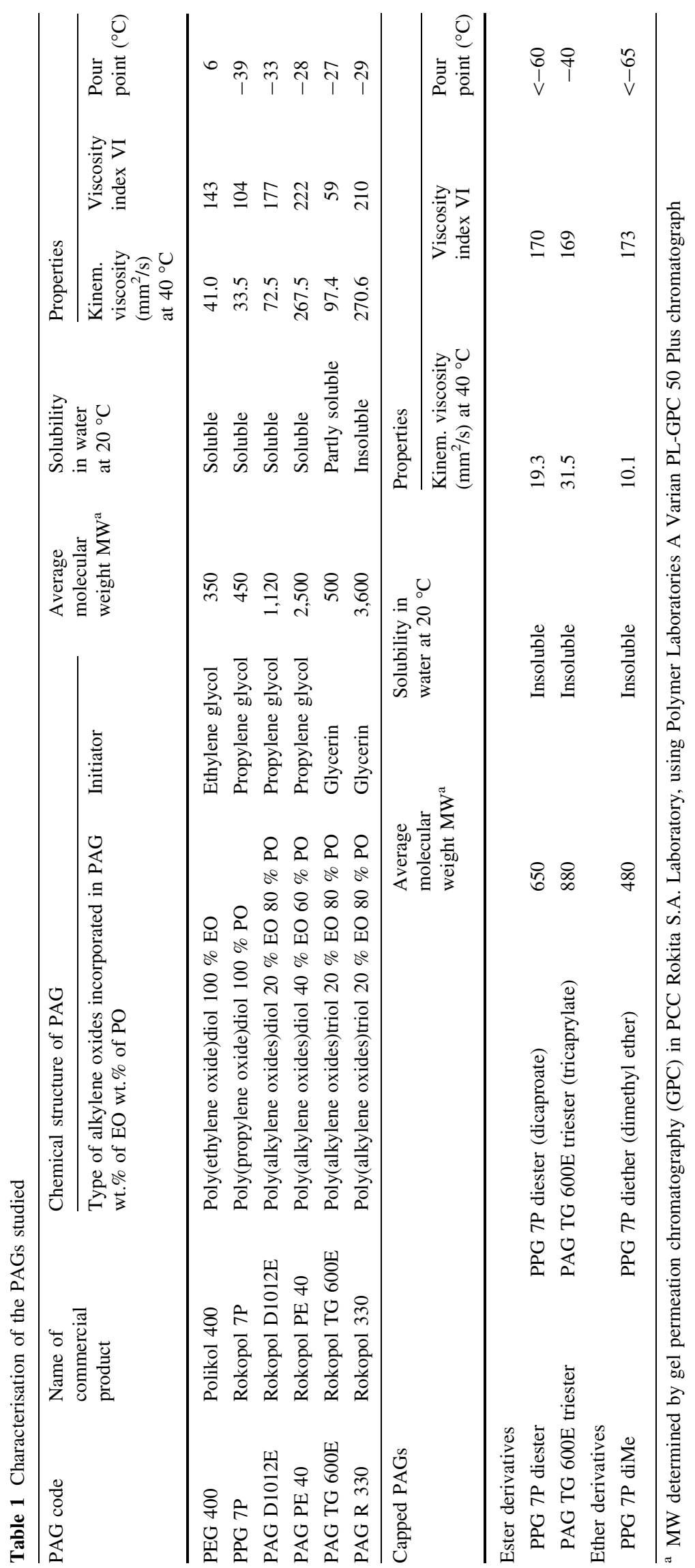


For comparative purposes, biodegradability tests were performed on the conventional mineral base oil SAE 10/95 (obtained from Rafineria Gdańska S. A., Poland), characterised by a kinematic viscosity of $34.5 \mathrm{~mm}^{2} / \mathrm{s}$ (at $40{ }^{\circ} \mathrm{C}$ ), a viscosity index of 94 and a pour point of $-15^{\circ} \mathrm{C}$. The studied oil was obtained as a solvent-refined base oil and is composed of structurally variable hydrocarbons with an average molecular weight of $385 \mathrm{Da}$ (determined by VPO method in toluene at $45{ }^{\circ} \mathrm{C}$ using KNAUER vapour pressure osmometer). These hydrocarbons include $76 \mathrm{wt} . \%$ of saturated ones, namely isoparaffins and mono- and polynaphthenes with short and long paraffinic chains, and 24 wt. $\%$ of aromatics.

\section{Methods of PAG Biodegradability Testing}

\section{ISO 14593 Test ( $\mathrm{CO}_{2}$ Headspace Test)}

The ISO 14593 procedure [21] is used in the OECD 310 test [22] recommended, together with the tests of the OECD 301 A-F series, for ready biodegradability assessment. The method enables the evaluation of ultimate biodegradation under aerobic conditions (mineralisation to carbon dioxide) of an organic compound in an aqueous medium by measuring the increase in total inorganic carbon over time with respect to a blank to which the test substance has not been added. The ultimate biodegradability of the PAGs that were tested was evaluated by making use of the ISO 14593 procedures, where sealed serum bottles ( $600 \mathrm{ml}$ in volume) containing test solutions $(400 \mathrm{ml})$ were used as test vessels for incubation. In this way, a headspace with an air-to-liquid volume ratio of 1:2 was obtained. The test solutions (with concentrations varying between 20 and $25 \mathrm{mg} \mathrm{C} / \mathrm{L}$ ) were prepared by adding watch glasses ( $\varnothing 18 \mathrm{~mm}$ ) with weighed quantities of the test PAGs into the vessels that contained the mineral salts medium. Prior to the addition of the inoculum, the solutions were subjected to dispersion with ultrasound $\left(25 \mathrm{kHz}, 20{ }^{\circ} \mathrm{C}\right)$. The inoculum was a $1 \mathrm{ml}$ dose of activated sludge with a cell density of $10^{8} \mathrm{CFU} / \mathrm{ml}$ (colony forming units per $\mathrm{ml}$ ) collected from the aeration tank at the Wrocław Wastewater Treatment Plant in Janówek. The tests were conducted for 28 days. The test bottles (three items for each duration, $\mathrm{t}_{\mathrm{x}}$, of the test corresponding to 7 , 14, 21 and 28 days) were incubated in the dark at $22 \pm 2{ }^{\circ} \mathrm{C}$, by using water-bath orbital bio-shakers (150 rpm, amplitude 5). The quantity of the total inorganic carbon (TIC) produced after time $t_{x}$ of biodegradation was calculated in terms of the $\mathrm{CO}_{2}$ concentration in the headspace gas that was taken for chromatographic analysis (Perkin Elmer Autosystem XL Arnel M 208, equipped with a thermal conductivity detector) after $\mathrm{pH}$ adjustment (with orthophosphoric acid) in the medium to values lower than
3. The percentage of biodegradation was calculated from the quantity of the TIC evolved in the PAG-containing test vessel (after subtracting the quantity of the IC evaluated in the blank vessel) in relation to the theoretical amount of inorganic carbon (ThIC) that was calculated based on the average molecular weight, the chemical structure of the tested PAG and the amount of the test compound that was initially added. Aniline was used as the reference substance in order to verify the activity of inoculum.

\section{ISO 14593 Test with Pre-exposed Activated Sludge Inoculum}

The procedure of the ISO 14593 test is used in both the OECD 310 test and the CONCAWE test [23], which was proposed as a draft of the OECD 302D test [24]. The main difference between the CONCAWE and OECD 310 tests is in the use of a pre-exposed inoculum in the former to give a test that assesses inherent biodegradability.

To examine how the adaptation of the activated sludge inoculum contributes to the enhancement of the inoculum's capacity for the biodegradation of PAGs whose structure consists of polymers with polyoxypropylene chains, tests were performed according to the procedure of ISO 14593 with a pre-exposed inoculum. The pre-exposure of the activated sludge inoculum was performed in the presence of pooled PPG 7P and PAG TG 600E according to the method described by Battersby et al. [23] and OECD 302D (draft) [24]. In our laboratory, the pre-exposure procedure was carried out by using replicate Erlenmeyer flasks of a $500 \mathrm{ml}$ volume. Each flask contained $250 \mathrm{ml}$ of mineral salts medium (prepared according to ISO 14593) supplemented with $12.5 \mathrm{mg}$ of yeast extract and $1 \mathrm{ml}$ of activated sludge (collected from the aeration tank at the Wrocław Wastewater Treatment Plant in Janówek, as described previously). The replicate flasks (closed with foam stoppers that facilitated the exchange of gases) were incubated in the dark at $20 \pm 1{ }^{\circ} \mathrm{C}$ in a water-bath orbital bio-shaker (150 rpm, amplitude 5) for 14 days. On the 1 st day and thereafter on the 7 th and 11th day of incubation, each flask was supplied with a mixture of PPG 7P and PAG TG 600E in quantities corresponding to the carbon concentration in the medium, which were 4,8 and $8 \mathrm{mg} \mathrm{C} / \mathrm{L}$, respectively. On the 14th day of the procedure, the medium with the preexposed activated sludge inoculum was filtered through glass wool and aerated with $\mathrm{CO}_{2}$-free air for $24 \mathrm{~h}$ to reduce the content of organic carbon. PPG 7P and TG 600E biodegradability were assessed according to the method described in ISO 14593 by using the pre-exposed inoculum in amounts of $10 \% \mathrm{v} / \mathrm{v}$ in compliance with the OECD 302D draft (e.g., $40 \mathrm{ml}$ of inoculum was added to $360 \mathrm{ml}$ of mineral salts medium). 
ISO 9888 Test (Zahn-Wellens Method) with Modified Procedure

The procedure of the ISO 9888 test [25] is similar to the method described in OECD 302 B [26], which enables the inherent biodegradability of water-soluble organic compounds to be evaluated by analyzing dissolved organic carbon (DOC) reduction or measuring chemical oxygen demand (COD) variations in the test solutions. As this test was designed for soluble substances, the test for poorly water-soluble substances (such as the PAG TG600E and PAG R330 that were tested) required the application of our own, appropriately modified method for the preparation of the test vessels [27]. The primary objective of the modification was to reduce the errors resulting from the insufficient size of the samples, which failed to represent the mean concentrations of the test PAGs in aqueous dispersion.

The tests were conducted in $250 \mathrm{ml}$ Erlenmeyer flasks that had been prepared for periods of $3.5 \mathrm{~h}$ (at the beginning of the test), 7, 14, 21 and 28 days. For each period, there were triplicate flasks that contained the PAG tested, triplicate flasks with ethylene glycol as the reference substance, and duplicate blank flasks. Each flask contained $150 \mathrm{ml}$ of the dispersion tested (with a test compound concentration of $200 \mathrm{mg} / \mathrm{l}$ ), which was obtained by adding watch glasses with weighed quantities of the test PAGs into the flasks that contained the mineral salts medium. Prior to inoculation with $10 \mathrm{ml}$ of activated sludge (with a cell density of $10^{8} \mathrm{CFU} / \mathrm{ml}$ collected from the aeration tank at the Wrocław Wastewater Treatment Plant in Janówek), the solution was subjected to ultrasonic dispersion $(25 \mathrm{kHz}$, $20{ }^{\circ} \mathrm{C}$ ) according to ISO 10634 [28]. During the test, the flasks were incubated in the dark at $20 \pm 1{ }^{\circ} \mathrm{C}$ in a water-bath orbital bio-shaker (150 rpm, amplitude 4).

After a defined incubation time $\left(\mathrm{t}_{\mathrm{x}}\right)$, the aqueous medium in the flask underwent ultrasonic dispersion followed by filtration. Two samples of $50 \mathrm{ml}$ volume were then collected for COD determination in accordance with the ISO 6060 method [29]. The extent of biodegradation is expressed as the percentage of COD reduction and was calculated as the ratio of the mean COD value obtained from the analysis of each test flask $\left(\mathrm{t}_{\mathrm{x}}\right)$ incubated with the PAG-toCOD value determined at the beginning of the test $\left(t_{o}\right)$. Each COD value was additionally corrected by subtracting the appropriate value of COD determined for the blank.

\section{Results and Discussion}

Ultimate Biodegradability of Oily Fluids with PAG Structures

The poly(alkylene glycol) polymers that were studied (Table 1) were chosen to represent PAGs differing in their chemical structures and having average molecular weights that varied between 350 and 3,600 Da. They are oily fluids with kinematic viscosities ranging from 30 to $270 \mathrm{~mm}^{2} / \mathrm{s}$ (at $40{ }^{\circ} \mathrm{C}$ ), a high viscosity index and a low pour point. Owing to these properties, homopolymers of propylene oxide and copolymers of propylene oxide and ethylene oxide are used not only as raw materials for industrial applications but also as synthetic base oils for the production of lubricants. This does not hold true for poly(ethylene glycol)s, mainly because of their excessively high pour points. PEG 400, a product with an average molecular weight of $350 \mathrm{Da}$, was included in the tests for comparative purposes because PEG biodegradability is commonly described in the literature [11, 13-17].

As seen in Table 1, the physicochemical properties and water solubility of the PAGs depend on their average molecular weight and chemical structure, which result from the proportion of ethylene oxide and propylene oxide used for the synthesis or from the structure of the polymer backbone (linear vs. branched) (Fig. 1). It was therefore possible to demonstrate the correlation between the properties and the defined characteristic structural features of the PAGs. An essential objective of the studies undertaken in this work was to assess how the structure of the PAG affects its biodegradability.

The results of biodegradability studies for all the PAGs, with the exception of capped PAGs, by the use of ISO 14593 are shown in Fig. 2. The progress of the aerobic biodegradation process during the 28-day test with non-preexposed inoculum is shown in relation to aniline, which was used to validate the proper course of the test. The lag phases in the biodegradation of two compounds, namely PPG 7P and PAG TG 600E, were obtained. Therefore, PPG 7P and PAG TG 600E were additionally studied in the test with pre-exposed (adapted) inoculum. As expected, PEG 400, being a homopolymer of ethylene oxide and used as a positive control, was most readily biodegraded (the ultimate biodegradation value determined at the end of the test was $77 \%$ ). PPG 7P, being a homopolymer of propylene oxide, and PAG TG 600E, being a copolymer, are both of similar molecular weight and are degraded to a slightly lower extent (64 and $68 \%$, respectively).

Even though the ISO 14593 test does not contain such a term as "ready biodegradability", the conditions and procedures included there are identical with those of the OECD 310 test [22], which makes it possible to establish whether or not the substance being examined is readily biodegradable. Thus, from the analysis of the biodegradation curve (Fig. 2), we infer that PEG 400 can be considered as a substance of ready biodegradability because it meets two important criteria: the pass level in the $\mathrm{CO}_{2}$ evolution is higher than $60 \% \mathrm{TIC} / \mathrm{ThIC}$, and this level has been reached within the 28-day test period by the end 
Fig. 2 Aerobic biodegradation curves of PAGs, mineral oil and aniline (reference substance) determined in the ISO 14593 test, with non-pre-exposed inoculum (multiplication symbol; open square; open triangle; open circle; open diamond) and pre-exposed inoculum (filled square; filled triangle); standard deviation from three repetitions (a)

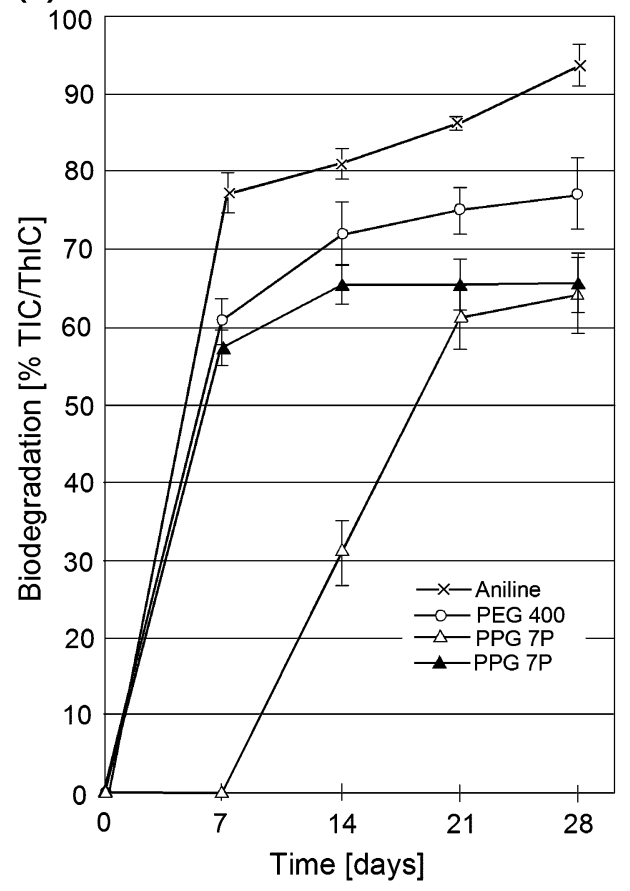

(b)

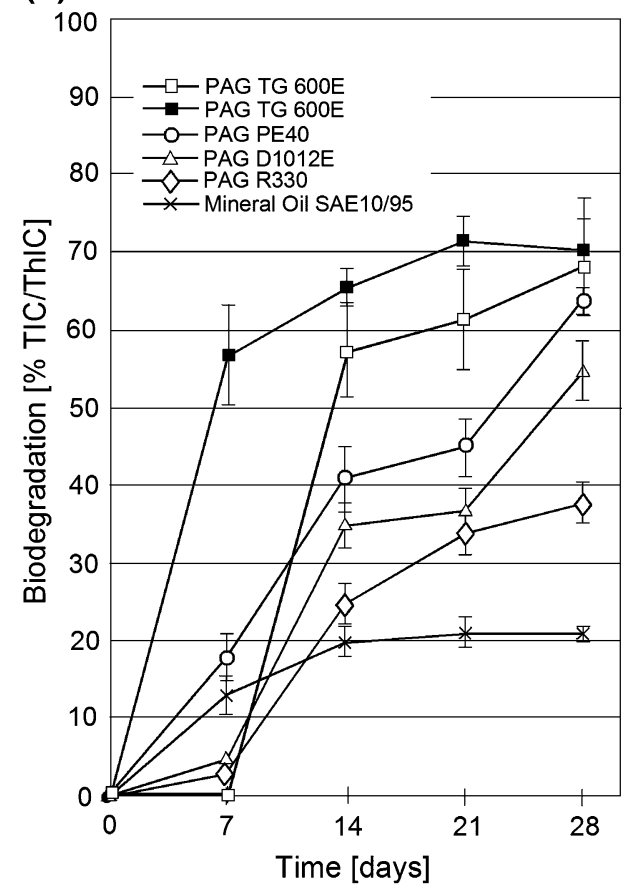

of the 10-day window, which begins when biodegradation reaches $10 \%$.

The PPG 7P and PAG TG 600E biodegradation curves determined by the ISO 14593 test (with non-pre-exposed inoculum) demonstrate lack of progress in their ultimate biodegradation to the 7th day of the test. After the 7th day, rapid progress in biodegradation was observed for PAGs whose ultimate biodegradation level by the end of the test exceeded $60 \%$. The high extent of ultimate biodegradation achieved in the ISO 14593 test for PPG 7P and PAG TG $600 \mathrm{E}$ indicates that both the fluids readily undergo aerobic biodegradation in an aqueous medium even though they fail to fulfil the 10-day window criterion.

The results of PEG biodegradability are consistent with those reported by Bernhard et al. [16], but they differ from the results obtained by Zgola-Grześkowiak et al. [14], who assessed the relative rates of PEG and PPG biodegradation. These differences most likely result from the differences in applied analytical procedures.

The presence of 7-day lag phases for PPG 7P and PAG TG $600 \mathrm{E}$ degradation observed in our test substantiates the theory formulated by Kawai and Tachibana et al. [11, 12]. They state that PEG polymers are biodegraded by the micro-organisms of the activated sludge, which utilise constitutive enzymes, namely alcohol and aldehyde dehydrogenases; these enzymes have been identified in the bacteria of the activated sludge used in PEG biodegradation tests. They have also found that PEG-degrading microorganisms fail to degrade PPG. When degrading PPG, micro-organisms that colonise the activated sludge require adaptation to this polymer by induction of the desired enzymes, which are indispensable for the oxidation of PPG alcohol groups.

The observed lag phase in the biodegradation of PPG 7P and PAG TG 600E and their rapid biodegradation upon preexposed conditions strongly support the observations of Kawai and Tachibana. Thus, the ISO 14593 test involving a pre-exposed (adapted) inoculum demonstrates that, on the 7th day of the test, both PPG 7P and PAG TG 600E reached an extent of ultimate biodegradation higher than $50 \%$ and that, after the 14th and 21st day, respectively, a plateau was observed. It is essential to note that although the adaptation of the inoculum to the PAGs that were examined failed to increase the efficiency of biodegradation, it accelerated the occurrence of the plateau phase during the test.

As can be seen from the biodegradation curves of PAGs with a copolymer structure and an average molecular weight higher than 1,000 Da (Fig. 2b), the PAG PE 40 with a greater proportion of oxyethylene groups in its structure is biodegraded faster than the PAG D1012E copolymer with a higher proportion of oxypropylene groups. This is significant because PAG PE 40 has an average molecular weight that is two times higher. The extent of ultimate biodegradation achieved by these PAGs on the 28th day of the test totalled 64 and $55 \%$, respectively. Their biodegradation curves demonstrate a slowdown in the progress of the biodegradation process between the 14th and 21st days of the test. This suggests that, over this period, the inoculum is being adapted to the structures of the metabolites produced during the test. 
PAG $\mathrm{R}$ 330, a representative of the poly(alkylene oxides)triols, has an average molecular weight of 3,600 Da (the highest amongst the studied compounds) and, being of the highest viscosity, was biodegraded at the slowest rate. The rise in the extent of biodegradation and the lack of plateau at the end of the test suggests an inherent biodegradability of PAG R 330 in an aqueous medium under aerobic conditions. This finding is important, especially when the biodegradation curve for PAG R 330 is compared with the biodegradation curve of the poorly biodegradable mineral base oil SAE 10/95.

In Fig. 3, results of the biodegradability values obtained in the ISO 9888 test (Zahn-Wallens test) are presented. Figure 3 includes the biodegradation curve for ethylene glycol (the reference substance for assessing the correctness of the test) and the biodegradation curve for the mineral oil SAE 10/95. As shown by these plots, both the biodegradation curves and the biodegradation levels determined on particular days of the test differ from the results of the ISO 14593 test. However, both tests gave more or less the same results regarding classification of different PAGs according to their mineralisation (the highest was for PEG 400; the lowest, for PAG R 330). Despite the differences, the results of this test indicate that none of the PAGs examined (except PEG 400) reached the plateau phase in the biodegradation process. This finding substantiates the inherent biodegradability of PAGs in an aqueous medium under aerobic conditions.

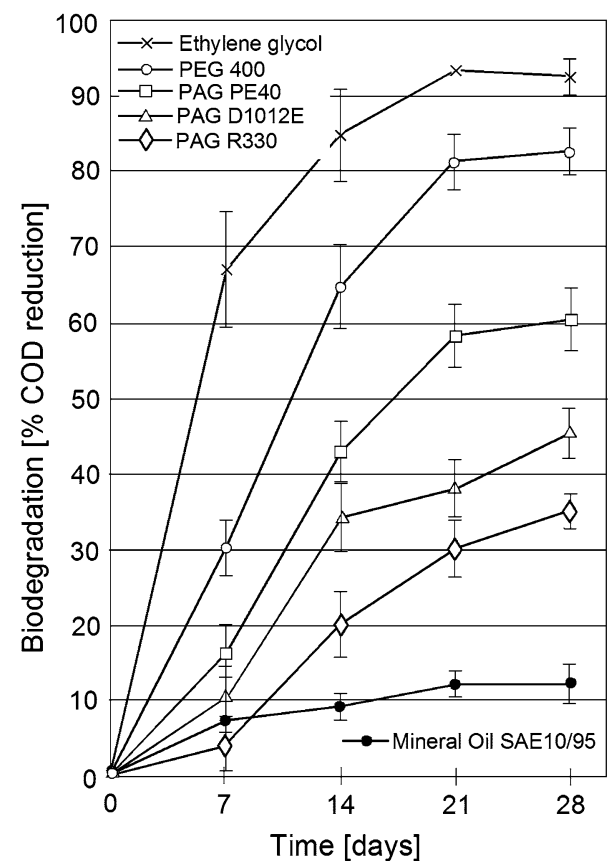

Fig. 3 Aerobic biodegradation curves of PAGs determined by the ISO 9888 test, with non-pre-exposed inoculum; standard deviation from three repetitions
Ultimate Biodegradability of Capped PAGs

As shown in Table 1, acylation or etherification of the terminal hydroxyl groups on the polymer backbone resulted in dramatic changes in the properties of the obtained capped PAGs. A previous study [19] has revealed that the differences between the properties of the PAGs and their acyl derivatives are attributable to the number of hydroxyl groups in the molecule and, consequently, to the proportion of the acyl groups incorporated into the PAG structure as a result of esterification.

The biodegradation curves for PPG 7P diester (dicaproate) and PAG TG 600E triester (tricaprylate) determined by ISO 14593 (Fig. 4) show a progress in their biodegradation on the 7th day of the test and a higher extent of their ultimate biodegradation as compared with PPG 7P and PAG TG 600E (Fig. 2). The extent of biodegradation for PPG 7P diester and PAG TG 600E triester on the 7th day of the test reached 29 and $41 \%$, respectively. Evidently, the biodegradation of the acyl derivatives of PAGs at the initial stage of the test is due to the enzymatic hydrolysis of the ester bonds and metabolic changes in the caproic or caprylic acid residues via the $\beta$-oxidation pathway. This seems to offer an explanation for the higher extent of biodegradation in the case of the triester derivative as compared with the extent of biodegradation in the case of the diester derivative. Moreover, the biodegradation curves reveal a slower progress in ultimate biodegradation between the 7 th and the 14th days

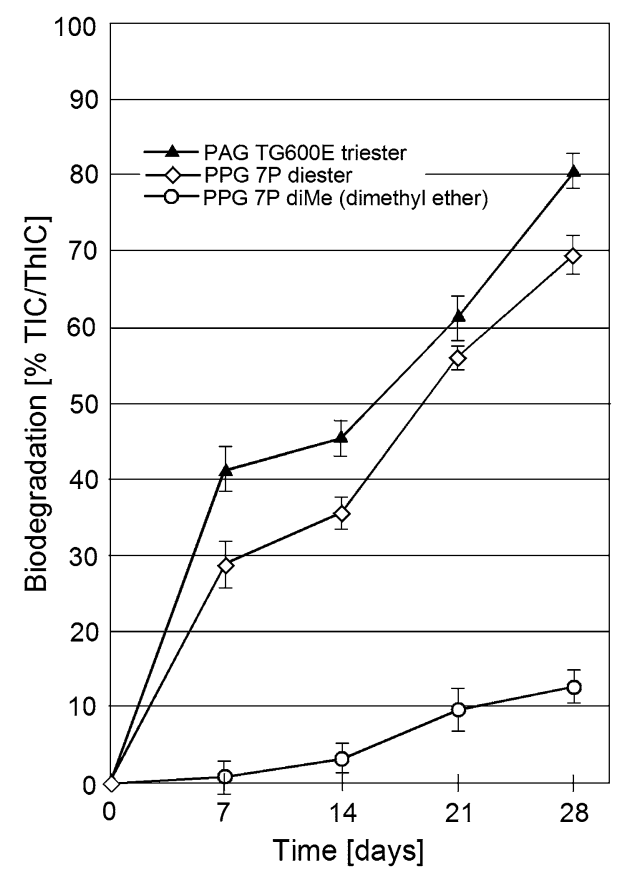

Fig. 4 Aerobic biodegradation curves of capped PAGs (ester or ether derivatives) determined in the ISO 14593 test, with non-pre-exposed inoculum; standard deviation from three repetitions 
of the test, followed by a rise in the rate of biodegradation after the 14th day. These findings imply that, between the 7 th day and the 14th day, the micro-organisms of the sludge adapted to the metabolites produced by the hydrolysis of the esters, seemingly to the oxypropylene chains of the PAGs with terminal hydroxyl groups.

As seen from Fig. 4, the process of biodegradation of PPG 7P dimethyl ether (PPG 7P diMe) is negligible. This is in sharp contrast with the diester derivatives. Functionalisation of the hydroxyl moieties with ethers evidently leads to non-biodegradable PAGs. This is logic because ethers are unusually stable both chemically and biologically. Seemingly, this is an explanation for the poor biodegradability of the PAG-type oils indicated in some publications where they are taken for the purpose of comparison with other base oils [30]. As for the PAGs with one or two terminal hydroxyl groups, the test results substantiate their potential capacity for biodegradation under aerobic conditions in an aqueous medium [1].

In conclusion, the PAG backbones with terminal ester bonds favourably influence the biodegradation of these PAGs, whereas those with terminal alkyl ether groups prevent such biodegradation. This result also supports earlier findings that the presence of terminal hydroxyl group is a requisite for the biodegradation of the PEG chains $[11,18]$.

\section{Conclusions}

Homopolymers of propylene oxide and random copolymers of ethylene oxide and propylene oxide with average molecular weights of 350-3,600 Da belonging to the PAG polymers are identified as oily fluids with kinematic viscosities in the range of $30-270 \mathrm{~mm}^{2} / \mathrm{s}$ (at $40{ }^{\circ} \mathrm{C}$ ). PAGs of that type exhibit noticeably better ultimate biodegradability in an aqueous medium compared with the poorly biodegradable mineral oils.

The most significant factors that affect the biodegradability of the PAGs that were tested are the following:

- terminal hydroxyl groups or acyl groups in the polymer backbone of the PAG molecules are required for the commencement of metabolic changes;

- the lower the average molecular weight of the PAG, the faster the rate of their ultimate biodegradation in an aqueous medium;

- the greater the proportion of oxyethylene to oxypropylene groups in the structures of the PAGs, the more readily they are degraded;

- PAGs with an average molecular weight of up to 1,000 Da, including homopolymers of ethylene oxide, homopolymers of propylene oxide and copolymers of ethylene and propylene oxides, can be considered as readily biodegradable compounds;

- capped PAGs containing terminal ether groups are practically non-biodegradable. This indicates that the presence of hydroxyl group is indispensable for the start of the degradation process of the PAG backbone;

- The progress of the biodegradation of PAGs in aerobicaquatic environments depends not only on their chemical structures, but also on the type of the microorganisms participating in the metabolic changes. When poly(ethylene glycol)s are biodegraded, the microorganisms of the activated sludge most likely utilise constitutive enzymes of the alcohol and aldehyde dehydrogenase types, which lead to rapid progress of PEG biodegradation. When homopolymers or copolymers of propylene oxide are biodegraded, the microorganisms have to induce enzymes of the alcohol dehydrogenase type as well as other kinds of enzymes required for the use of these PAGs as carbon and energy sources by micro-organisms that are present in activated sludge. This extends the time of the commencement of PPG degradation by the period required for the adaptation of the micro-organisms to polyoxypropylene chains and results in the presence of a lag phase for the process. The use of inoculum pre-exposed to the PAGs that were examined fails to increase the efficiency of biodegradation, but it accelerates the occurrence of the plateau phase in biodegradability tests.

Acknowledgments The authors would like to thank PCC Rokita SA Poland for providing the polyalkylene glycol samples tested within the scope of this study. Thanks are also due to the management and staff of MPWiK Wrocław for the samples of activated sludge, which were used as inoculum. This work was financed by grant 344083/Z0306-W3 given by the Polish Ministry of Science and Higher Education.

Open Access This article is distributed under the terms of the Creative Commons Attribution License which permits any use, distribution, and reproduction in any medium, provided the original author(s) and the source are credited.

\section{References}

1. Matlock PL, Brown WL, Clinton NA (1999) Polyalkylene glycols. In: Rudnick LR, Shubkin RL (eds) Synthetic lubricants and highperformance functional fluids. Marcel Dekker Inc., New York

2. Baumgartner W (1998) Polyglycols-characteristics and applications. In: Bartz WJ (ed) Proceedings of the 11th international colloquium tribology, vol III. Technische Akademie Esslingen, Ostfildern, pp 2513-2526

3. Van Voorst R, Alam F (2000) Polyglycols as base fluids for environmentally friendly lubricants. J Synth Lubr 16:313-322

4. Lawford S (2006) Polyakylene glycols. In: Rudnick LR (ed) Synthetics, mineral oils, and bio-based lubricants: chemistry and technology. CRC/Taylor and Francis, Boca Raton 
5. Woydt M (2007) No/Low SAP and alternative engine oil development and testing. J ASTM Int 4(10). Paper ID JAI100898 available online at www.astm.org

6. Rudnick LR (2006) Synthetics, mineral oils, and bio-based lubricants: chemistry and technology. CRC/Taylor and Francis, Boca Raton

7. Bartz WJ (2006) Ecotribology: environmentally acceptable tribological practices. Tribol Int 39:728-733

8. Cain RB (1991) Biodegradation of Lubricants. In: Rossmoore HW (ed) 8th international biodeterioration and biodegradation. Elsevier Science Publisher, Amsterdam, pp 249-275

9. Goyan RL, Melley RE, Wissner PA, Ong WC (1998) Biodegradable lubricants. Lubr Eng 7:10-17

10. Battersby NS (2000) The biodegradability and microbial toxicity testing of lubricants-some recommendations. Chemosphere 41:1011-1027

11. Kawai F (2002) Microbial degradation of polyethers. Appl Microbiol Biotechnol 58:30-38

12. Tachibana S, Kuba N, Kawai F, Duine JA, Yasuda M (2003) Involvement of a quinoprotein (PQQ-containing) alcohol dehydrogenaze in the degradation of polypropylene glycols by the bacterium Stenotrophomonas maltophilia. FEMS Microbiol Lett 218:345-349

13. Huang Y-L, Li Q-B, Deng X, Lu Y-H, Liao X-K, Hong M-Y, Wang Y (2005) Aerobic and anaerobic biodegradation of polyethylene glycols using sludge microbes. Process Biochem 40: 207-211

14. Zgola-Grześkowiak A, Grześkowiak T, Zembrzuska J, Łukaszewski Z (2006) Comparison of biodegradation of poly(ethylene glycol)s and poly(propylene glycol)s. Chemosphere 64:803-809

15. Pan L, Gu J-D (2007) Characterization of aerobic bacteria involved in degrading polyethylene glycol (PEG)-3400 obtained by plating and enrichment culture techniques. J Polym Environ 15:57-65

16. Bernhard M, Eubeler JP, Zok S, Knepper TP (2008) Aerobic biodegradation of polyethylene glycols of different molecular weights in wastewater and seawater. Water Res 42:4791-4801

17. Marchal R, Nicolau E, Ballaguet J-P, Bertoncini F (2008) Biodegradability of polyethylene glycol 400 by complex microfloras. Int Biodeterior Biodegrad 62:384-390

18. Corti A, D'Antone S, Solaro R, Chiellini E (1998) Degradation of poly(ethylene glycol)—based nonionic surfactants by different bacterial isolates from river water. J Environ Polymer Degrad 6:121-131

19. Beran E (2003) Structurally modified polyglycols as biodegradable base stocks for synthetic lubricants. J Synth Lubr 20:3-14

20. Janik R, Matyschok H, Rybak WK, Ziółkowski JJ (1991) Synthesis of dimethyl ethers of polyoxyalkylene glycols-the sulphur dioxide absorbents. Polish J Chem 35:247-257

21. ISO Standard 14593 (1999) Water quality-evaluation of ultimate aerobic biodegradability of organic compounds in aqueous medium - method by analysis of inorganic carbon in sealed vessels $\left(\mathrm{CO}_{2}\right.$ headspace test). International Organization for Standardization

22. OECD Guidelines for Testing of Chemicals (2006) 310 ready biodegradability: $\mathrm{CO}_{2}$ in sealed vessels (Headspace Test). Organization for Economic Co-operation and Development, Paris

23. Battersby NS, Ciccognani D, Evans MR, King D, Painter HA, Peterson DR, Starkey M (1999) An inherent biodegradability test for oil products. Description and results of an international ring test. Chemosphere 38:3219-3235

24. OECD Guidelines for Testing of Chemicals (2001) Draft document. proposal for a new guideline 302D inherent biodegradability-CONCAWE test. Organization for Economic Cooperation and Development, Paris

25. ISO Standard 9888 (1999) Water quality—evaluation of ultimate aerobic biodegradability of organic compounds in aqueous medium-static test (Zahn-Wellens method). International Organization for Standardization

26. OECD Guidelines for Testing of Chemicals (1992) 302B inherent biodegradability: Zahn-Wellens/EMPA test. Organization for Economic Co-operation and Development, Paris

27. Beran E (2008) Experience with evaluating biodegradability of lubricating base oils. Tribol Int 41:1212-1218

28. ISO Standard 10634 (1995) Water quality-guidance for preparation and treatment of poorly water-soluble organic compounds for the subsequent evaluation of their biodegradability in an aqueous medium. International Organization for Standardization

29. ISO Standard 6060 (1989) Water quality-determination of chemical oxygen demand. International Organization for Standardization

30. Bartz WJ (1998) Lubricants and the environment. Tribol Int 31: $35-47$ 